\title{
Biomassa e extração de quitina e quitosana a partir de isolados de Cunninghamella sp.
}

\section{Biomass and chitin and chitosan extraction from Cunninghamella sp. Isolates}

\author{
Jeane Cândida Lopes ${ }^{1}$, Aloisio Freitas Chagas Junior ${ }^{2}$, Gessiel Newton Scheidt ${ }^{3}$, Layssah Passos \\ Soares $^{4}$, Lillian França Borges Chagas ${ }^{5}$
}

\begin{abstract}
Resumo
$\overline{\mathrm{O} \text { estudo objetivou avaliar a produção de biomassa de nove isolados de Cunninghamella sp. e da cepa }}$ referência de Cunninghamella elegans (CBMAI 0843) e estabelecer a capacidade de produção de quitina e quitosana por estas cepas. Assim como, caracterizar a quitosana fúngica obtida por parâmetros como massa molar, grau de desacetilação e distribuição dos grupos funcionais ao longo da cadeia polimérica. Para a maioria das cepas avaliadas, o período de maior crescimento foi em 48 horas de cultivo, sendo que, neste período, o isolado UFT Ce08 apresentou a maior quantidade de biomassa, 20,17 g L-1. Os rendimentos de quitina ficaram entre 15,64 a $30,33 \%$ e os rendimentos de quitosana entre 0,94 a 7,43\%. A cepa UFT Ce11 apresentou o melhor quantitativo de quitina e a cepa UFT Ce09, mesmo apresentando o segundo menor quantitativo de biomassa, $9,34 \mathrm{~g} \mathrm{~L}^{-1}$, teve o melhor rendimento de quitosana. Sete cepas isoladas no presente estudo apresentaram maior rendimento de quitosana comparada à cepa referência. O grau médio de desacetilação foi de $83,7 \%$ para quitosana obtida do isolado UFT Ce09 e $80,5 \%$ para quitosana obtida da cepa referência. As massas molares para a quitosana do isolado UFT $\mathrm{Ce} 09$ e da cepa referência foram de 43,031 e 19,215 $\mathrm{g} \mathrm{mol}^{-1}$, respetivamente. A espectrometria de infravermelho apresentou bandas com comprimentos de onda e grupos funcionais coincidentes com a literatura e com a quitosana comercial. A quitosana fúngica deste estudo apresentou propriedades que atestam sua qualidade e características de interesse biotecnológico e comercial.
\end{abstract}

Palavras chave: Cultivo submerso. Biopolímeros. Rendimento.

\begin{abstract}
The aim of this study was to evaluate the biomass production of nine Cunninghamella sp. isolates as well as by the reference strain Cunninghamella elegans (CBMAI 0843) and establish the chitin and chitosan production capacity by these strains. For most of the tested strains, the highest growth period was within 48 hours of cultivation, although the UFT Ce 08 isolate showed the highest amount of biomass, with $20.17 \mathrm{~g} \mathrm{~L}^{-1}$. Chitin yields were between 15.64 to $30.33 \%$ and chitosan yields were between 0.94 to $7.43 \%$. The UFT Ce11 strain presented the best chitin quantity and UFT Ce09 strain, even with the second smallest biomass quantity, had the best chitosan yield. This means that seven isolated strains in this study showed higher chitosan yield compared to the reference strain. The degree of deacetylation was $83.7 \%$ for the chitosan obtained from the UFT Ce09 isolate and $80.5 \%$ for the chitosan obtained from the reference strain. The chitosan molecular weight for the UFT Ce09 isolate and the reference strain were $43.031 \mathrm{~g} \mathrm{~mol}^{-1}$ and $19.215 \mathrm{~g} \mathrm{~mol}^{-1}$, respectively. The infrared spectroscopy presented bands with wavelengths and functional groups coincident to the literature and to the commercial chitosan. The fungal chitosan of this study showed properties that confirm its quality and characteristics of biotechnological and commercial interest.
\end{abstract}

Keyword: Submersed culture. Biopolymers. Yield.

\footnotetext{
${ }^{1}$ Mestrado em Biotecnologia pela Universidade Federal do Tocantins. Universidade Federal do Tocantins; Gurupi, Tocantins, Brasil.

2 Doutorado em Biotecnologia pela Universidade Federal do Amazonas. Universidade Federal do Tocantins; Gurupi, Tocantins, Brasil. E-mail: chagasjraf@uft.edu.br

${ }^{3}$ Doutorado em Processos Biotecnológicoas pela Universidade Federal do Paraná. Universidade Federal do Tocantins; Gurupi, Tocantins, Brasil.

${ }^{4}$ Graduanda em Engenharia Agronômica pela Universidade Federal do Tocantins. Universidade Federal do Tocantins; Gurupi, Tocantins, Brasil.

${ }^{5}$ Doutorado em Produção Vegetal pela Universidade Federal do Tocantins. Universidade Federal do Tocantins; Gurupi, Tocantins, Brasil.
} 
Lopes, J. C. et al.

\section{Introdução}

Presente na parede celular de fungos e no exoesqueleto de insetos e crustáceos, a quitina é o segundo polissacarídeo mais encontrado na natureza depois da celulose. Sua estrutura química é formada por longa cadeia linear de sucessivas unidades de amino monossacarídeo denominado de $\mathrm{N}$-acetilglicosamina (VÁZQUEZ et al., 2013).

A quitina apresenta um copolímero derivado da sua desacetilação parcial, a quitosana. Ambas têm sido extensamente estudadas devido ao potencial promissor nas áreas farmacêuticas, alimentícias e biomédicas (CHIEN; YEN; MAU, 2016; CROFTON et al., 2016; THARANATHAN; KITTURE, 2003).

A quitina e a quitosana são constituídas basicamente por unidades N-acetil-D-glicosamina e D-glicosamina unidas por ligações glicosídicas $\beta$ (1-4), em proporções variáveis, sendo que, a quitina, por conter acima de $40 \%$ de N-acetil-D-glicosamina, é insolúvel em solução aquosa de ácido acético 1\%, e a quitosana, composta, predominantemente, por unidades D-glicosamina, é um derivado solúvel (FRÁGUAS et al., 2015; SILVA; SANTOS; FERREIRA, 2006).

O grau médio de acetilação é o parâmetro empregado para caracterizar o conteúdo médio de unidades N-acetil-D-glicosamina da quitosana, que irá diferenciar os polímeros de quitosana em relação as suas propriedades físico-químicas, como solubilidade, pKa e viscosidade (SINGLA; CHAWLA, 2001). Em valores, as propriedades físico-químicas da quitosana como grau de N-desacetilação, estão em torno de 60 a $100 \%$ e a massa molar em torno de 3,8 a $20 \mathrm{kDa}$ (GAVHANE; GURAV; YADAV, 2013).

$\mathrm{O}$ interesse comercial nas aplicações de quitosana aumentou nas últimas três décadas, entretanto, apesar da enorme potencialidade, as aplicações de quitosana não vêm sendo implementadas na extensão esperada (CAMPANA-FILHO et al., 2007).

A carapaça de crustáceos é a fonte de quitina e quitosana mais utilizada, porém, o processo de extração destes polímeros utiliza a desacetilação termoquímica que acarreta desvantagens a nível econômico e ambiental (FRANCO et al., 2004; SANTOS et al., 2003).

Rendimentos iguais ou superiores de quitina e quitosana têm sido relatados utilizando fungos como fonte alternativa em comparação com fontes tradicionais. Recentes estudos ainda destacam métodos de otimização para obtenção de quitina e quitosana a partir da massa micelial do fungo Cunninghamella elegans (BERGER et al., 2016; FREITAS et al., 2006; TAYEL et al., 2016).

Uma estimativa da quantidade de quitina e quitosana é usualmente alcançada medindo-se os grupos $\mathrm{N}$-acetil glicosamina e glicosamina, na parede celular de fungos filamentosos. Essa quantidade corresponde a aproximadamente, 32,7 e $9,4 \%$ de quitina e quitosana respectivamente, e na casca de crustáceos o conteúdo de quitina varia entre 15 a 20\% (KARIMI; ZAMANI, 2013; PINTO; BETTIOL; MORANDI, 2010).

A biomassa de fungos do gênero Cunninghamella sp. tem sido intensamente estudada devido sua elevada quantidade de quitina e quitosana em sua parede celular, apresentando rendimentos entre 5 e $8 \%$ de quitosana, e em torno de 13 a $24 \%$ de quitina (FRANCO et al., 2005). Assim, métodos de otimização que visam implementar a produção destes biopolímeros, particularmente por esta classe de fungos, têm se destacado atualmente (BERGER et al., 2009, 2014; STAMFORD et al., 2007).

A utilização da biomassa fúngica para extração destes biopolímeros apresenta vantagens como: independe de fatores sazonais, produz em larga escala, extrai simultaneamente quitina e quitosana, o processo de extração é simples e de baixo custo e evita reações alérgicas a proteínas de crustáceos (STAMFORD et al., 2007; ZAMANI, 2010). Assim, avaliar a qualidade da quitosana extraída da parede celular fúngica viabiliza a possibilidade de comercializar este polímero extraído através de fontes alternativas.

Nosso objetivo foi avaliar a produção de biomassa de isolados de Cunninghamella sp. e da cepa referência de Cunninghamella elegans (CBMAI 0843) e estabelecer a capacidade de produção de quitina e quitosana por estas cepas, assim como, caracterizar a quitosana fúngica obtida por parâmetros como massa molar, grau de desacetilação e distribuição dos grupos funcionais ao longo da cadeia polimérica

\section{Material e Método}

\section{Microrganismos e condições de cultivo}

Utilizou-se amostras de nove cepas de Cunninghamella sp. isoladas de solos de áreas de cultivo. Para a coleta do solo foram selecionadas áreas cultivadas no município de Gurupi (TO, latitude $11^{\circ} 43^{\prime} 45^{\prime \prime}$ sul e longitude $49^{\circ} 04^{\prime} 07^{\prime \prime}$ oeste) com altitude de $287 \mathrm{~m}$ e área de $1846,6 \mathrm{~km}^{2}$ e no município de Formoso do Araguaia (TO, latitude: $11^{\circ} 47^{\prime} 48^{\prime \prime}$ sul e longitude: $49^{\circ} 31^{\prime} 44^{\prime \prime}$ oeste) com altitude de $240 \mathrm{~m}$ e área de $13510,5 \mathrm{Km}^{2}$. 
As coletas do solo no município de Gurupi foram realizadas em locais próximos da Universidade Federal do Tocantins (UFT) abrangendo áreas experimentais de cultivo de milho, soja e mandioca, irrigadas constantemente. No município de Formoso do Araguaia foram coletadas amostras nas áreas experimentais de cultivo de arroz.

Foram coletadas um total de 50 amostras de solo com três repetições cada, sub amostras distantes em 3 pontos entre si, respeitando raio de $1 \mathrm{~m}$, retiradas de 0 a $15 \mathrm{~cm}$ de profundidade. O método de espalhamento de solo, descrito inicialmente por Duddington (1951) e modificado por Santos, Ferraz, Muchovej (1991), foi utilizado para o isolamento do fungo.

A caracterização morfológica foi realizada no laboratório de microbiologia da UFT Campus Gurupi, procedida através da observação das características macroscópicas das colônias como micélio cotonoso, hifas brancas altas entrelaçadas delicadas, de fácil ruptura, características comuns a todas espécies do gênero Cunninghamella (ALEXOPOULOS; MIMS; BLACKWELL, 1996; CAMPBELL; JOHNSON, 2013). Foi feita a comparação macroscópica de cada isolado com a cepa de referência, de modo a estabelecer semelhanças.

Para identificação microscópica dos fungos, preparou-se lâminas de microscopia com o material a fresco, examinando-as em microscópio óptico com objetiva de 40x. Buscou-se observar esporangióforos eretos, simples ou ramificados com a extremidade contendo vesículas globosas (esporangióforos) nas quais estão inseridos os esporangiósporos, micélio não septado e esporos hialinos, unicelulares, globosos, com paredes lisas ou com espículas, tal como destacam Barnett e Hunter (1999); Pitt e Hocking (2009). Procedendo-se então a classificação em nível de gênero das cepas isoladas.

Os isolados identificados foram depositados no laboratório de microbiologia da Universidade Federal do Tocantins (UFT), Campus Gurupi. Sendo eles UFT Ce02, UFT Ce04, UFT Ce05, UFT Ce06, UFT Ce08, UFT Ce09, UFT Ce11, UFT Ce14 e UFT Ce20, e amostra da cepa referência de Cunninghamella elegans (CBMAI 0843) mantidas a $4^{\circ} \mathrm{C}$ em placas de Petri contendo meio BDA (Batata, Dextrose, Ágar). Suspensões de esporos foram preparadas e padronizadas para uma concentração final de $10^{6}$ esporos $\mathrm{mL}^{-1}$, através da contagem de esporos em hematocitômetro.
Para o cultivo submerso, foram inoculados $1 \mathrm{~mL}$ de cada suspensão de esporos em frascos Erlenmeyer de $250 \mathrm{~mL}$, contendo $50 \mathrm{~mL}$ do meio YPD $(02 \%$ de extrato de levedura, $1 \%$ de peptona e $2 \%$ de Dextrose, $\mathrm{pH}$ ajustado para 4,5). Procedeu-se, então, a incubação a temperatura ambiente, durante 120 horas sob agitação orbital constante de $100 \mathrm{rpm}$, em mesa agitadora orbital. Os experimentos foram realizados com quatro repetições.

A cada 24 horas retirou-se da agitação frascos Erlenmeyer contendo a biomassa micelial de cada amostra fúngica e filtrou-se a biomassa desprezando o meio líquido. A biomassa foi submetida a processo de secagem em estufa a $50^{\circ} \mathrm{C}$. Em seguida, a massa micelial seca foi triturada a pó e pesada em balança analítica.

\section{Extração de quitina e quitosana}

O processo geral envolveu a desproteinização da biomassa com $2 \% \mathrm{~m} / \mathrm{v}$ de solução de hidróxido de sódio $\left(30: 1 \mathrm{v} / \mathrm{m}, 90^{\circ} \mathrm{C}, 2 \mathrm{~h}\right)$; a separação da fração insolúvel em álcali (FIA) por centrifugação (4.000 rpm, 15 min); a extração da quitosana a partir da FIA com ácido acético $10 \% \mathrm{v} / \mathrm{v}\left(40: 1 \mathrm{v} / \mathrm{m}, 60^{\circ} \mathrm{C}, 6 \mathrm{~h}\right)$; a separação da quitina por centrifugação $(4.000 \mathrm{rpm}, 15$ $\min$ ); a precipitação da quitosana a partir do extrato, a pH 9,0 ajustado com solução de $\mathrm{NaOH} 4 \mathrm{M}$; a lavagem da quitina e quitosana com água, etanol e acetona e secagem ao ar a $20^{\circ} \mathrm{C}$ (FRANCO et al., 2004).

\section{Caracterização da quitosana}

A quitosana extraída da cepa referência e do isolado que apresentou maior rendimento, foi caracterizada quanto ao grau médio de desacetilação, a massa molar viscosimétrica média e a distribuição dos grupos funcionais ao longo da cadeia polimérica.

O grau médio de desacetilação, definido como o número de grupos amino em relação aos grupos amida da cadeia polimérica (SANTOS et al., 2003), foi determinado através da titulação potenciométrica das soluções aquosas de quitosana. Assim, foram tomados $50 \mathrm{mg}$ de amostra dissolvidos em $50 \mathrm{~mL}$ de água destilada. $\mathrm{O}$ pH da solução foi ajustado para $\mathrm{pH}<2$ com adição de $\mathrm{HCl}$ concentrado. A solução após agitação por 20 horas foi titulada com uma solução aquosa de $\mathrm{NaOH} 0,1 \mathrm{~mol} \mathrm{~L}^{-1}$, previamente padronizada (ABREU; CAMPANA-FILHO, 2005; CZECHOWSKA-BISKUP et al., 2012). Os valores de $\mathrm{pH}$ foram registrados utilizando um $\mathrm{pHmetro}$ digital. A 
solubilização e a titulação foram realizadas em banho termostatizado a $25,0 \pm 0,1^{\circ} \mathrm{C}$.

O grau de desacetilação (GD) foi calculado a partir do volume de $\mathrm{NaOH}$, utilizando a fórmula $\% \mathrm{GD}=[\mathrm{M}(\mathrm{V} 2-\mathrm{V} 1) \times 161] / \mathrm{W} \times 100$, em que V1 é o volume de base usado na neutralização de $\mathrm{HCl} \mathrm{em}$ excesso, expresso em mL; V2 é o volume de $\mathrm{NaOH}$ correspondente à neutralização dos grupos amino presentes no polímero, expresso em $\mathrm{mL}$; $\mathrm{M}$ é a concentração da solução de $\mathrm{NaOH}$ e W é a massa da quitosana em mg. $\mathrm{O}$ valor 161 corresponde à massa equivalente a um monômero do polímero.

Para determinação de viscosidade, utilizou-se um viscosímetro de tubo em $\mathrm{U}$, de vidro transparente, de $10 \mathrm{~mL}$ e banho termostatizado em $25,0 \pm 0,1^{\circ} \mathrm{C}$, e procedeu-se conforme a metodologia descrita por Santos et al. (2003).

A viscosidade intrínseca, obtida pela extrapolação gráfica da relação de viscosidade reduzida versus concentração, foi utilizada para determinar a massa molar viscosimétrica média $\left(\mathrm{M}_{\mathrm{V}}\right)$ a partir da equação de Mark-Houwink-Sakurada, $[\mathrm{n}]=\mathrm{KM}_{\mathrm{v}}{ }^{\alpha}$, em que [n] é a viscosidade intrínseca; $\mathrm{K}$ e $\alpha$ são constantes para um determinado sistema de polímero/solvente/ temperatura, neste caso $\mathrm{K}=0,075$ e $\alpha=0,76$ e $\mathrm{M}_{\mathrm{V}}$ é a Massa molar viscosimétrica média (KASAAI, 2007).

Os espectros na região do infravermelho foram registrados em um espectrofotômetro infravermelho com transformação de Fourier Agilent Cary 360, na região de 400 a $4.000 \mathrm{~cm}^{-1}$, utilizando amostras de quitina e quitosana previamente secas em estufas à temperatura de $40^{\circ} \mathrm{C}$. A amostra foi prensada em prensa hidráulica para formar uma pastilha de aproximadamente $0,20 \mathrm{~mm}$ de espessura, em seguida foi analisada.

\section{Análise estatística}

Os dados da quantificação de biomassa foram submetidos à análise de variância, e as médias foram comparadas pelo teste de Scott-Knott a 5\% de significância, utilizando o programa estatístico Assistat 7.6. Para viscosidade foram realizadas análises de regressão e correlação.

\section{Resultados e Discussão}

\section{Produção de biomassa}

As massas miceliais coletadas durante as 120 horas de cultivo foram utilizadas para estabelecer o perfil de crescimento a partir da biomassa seca. Para a maioria das cepas avaliadas, o período de maior crescimento foi em 48 horas de cultivo, sendo que, neste período, o isolado UFT Ce08 apresentou quantidade de biomassa superior $(\mathrm{p}<0,05)$ com $20,17 \mathrm{~g} \mathrm{~L}^{-1}$, seguido do isolado UFT Ce20 com 12,64 $\mathrm{g} \mathrm{L}^{-1}$, cepa referência com 10,78 $\mathrm{g} \mathrm{L}^{-1}$, UFT Ce11 com 10,72 $\mathrm{g} \mathrm{L}^{-1}$, UFT Ce02 com 9,42 $\mathrm{g} \mathrm{L}^{-1}$, UFT Ce09 com 9,34 $\mathrm{g} \mathrm{L}^{-1} \mathrm{e}$ o isolado UFT Ce05 com menor biomassa, $9,15 \mathrm{~g} \mathrm{~L}^{-1}$.

Os isolados UFT Ce04 e UFT Ce14 apresentaram crescimento superior $(\mathrm{p}<0,05)$ no período de 72 horas com 13,26 e 10,50 $\mathrm{g} \mathrm{L}^{-1}$, respectivamente. Já o isolado UFT Ce06 teve crescimento superior $(\mathrm{p}<0,05)$ em 96 horas com 11,17 $\mathrm{g} \mathrm{L}^{-1}$ de biomassa seca (Tabela 1).

As cepas que apresentaram melhor crescimento, ou seja, maior biomassa, considerando todos os períodos de avaliação, foram os isolados UFT Ce08, UFT $\mathrm{Ce} 04$ e UFT Ce20. Já os isolados UFT Ce05, UFT $\mathrm{Ce} 02$ e UFT Ce09 apresentaram menor quantidade de biomassa, consequentemente, o pior crescimento (Tabela 1).

Para a maioria das cepas avaliadas, o período de maior crescimento foi em 48 horas, obtendo neste período o maior quantitativo de biomassa seca, 20,17 $\mathrm{g} \mathrm{L}^{-1}$. Stamford et al. (2007) também observaram crescimento exponencial em 48 horas, e, após este período, um declínio do crescimento, obtendo 20,4 g $\mathrm{L}^{-1}$ de biomassa ao cultivar C. elegans (UCP 542) em caldo de Jacatupé, resultado próximo ao do presente estudo.

Crescimento exponencial em todo período experimental foram relatados por Franco et al. (2004) e Souza (2004), ao cultivar C. elegans em meio sintético para mucolares, obtendo 11,6 e 122,9 mg $\mathrm{L}^{-1}$ de biomassa seca, respectivamente. Berger et al. (2009) também relataram crescimento crescente de $C$. elegans (UCP 542) por 96 horas de cultivo em meio $\mathrm{BD}$ (batata dextrose), porém, obtiveram apenas $6 \mathrm{~g} \mathrm{~L}^{-1}$ de biomassa seca.

Amorim et al. (2006) cultivando Cunninghamella bertholletiae (IFM 46.114) em meio YPD, observaram crescimento micelial contínuo em 120 horas, porém, a biomassa seca foi de apenas $9 \mathrm{~g} \mathrm{~L}^{-1}$.

Valores inferiores de biomassa de C. elegans (IFM 46109) em meio enriquecido com glicose, asparagina e tiamina, também foram relatados por Franco et al. (2005), com 11,6 $\mathrm{g} \mathrm{L}^{-1}$. Santos et al. (2013) também obtiveram valores menores de biomassa, $16,95 \mathrm{~g} \mathrm{~L}^{-1}$, em relação ao presente estudo, ao cultivar $C$. elegans (UCP/WFCC 0542) em meio composto por milhocina. 


\section{Rendimento de quitina e quitosana}

As cepas avaliadas apresentaram resultados heterogêneos no rendimento de quitina e quitosana. Os rendimentos de quitina ficaram entre 15,64 a $30,33 \%$ e os rendimentos de quitosana entre 0,94 a 7,43\% (Tabela 2). A cepa UFT Ce11 apresentou o maior quantitativo de quitina, $30,33 \%$, e o segundo melhor rendimento de quitosana, 5,91\%. A cepa UFT Ce09 apresentou maior quantidade de quitosana, $7,43 \%$, seguido pela cepa UFT Ce 20 com $5,55 \%$, UFT Ce0 8 com $4,66 \%$ e UFT Ce14 com $4,26 \%$.

A cepa UFT Ce 06 apresentou o menor rendimento de quitina e quitosana, $15,64 \%$ e $0,94 \%$, respectivamente. Já a cepa referência CBMAI 0843 teve o terceiro melhor rendimento de quitina, $24,29 \%$, porém, o terceiro menor rendimento de quitosana, $1,89 \%$, ou seja, sete cepas isoladas no presente estudo apresentaram maior rendimento de quitosana comparada à cepa referência (Tabela 2).

A cepa UFT $\mathrm{Ce} 09$ apresentou maior rendimento de quitosana, porém, menor crescimento micelial. Fukuda et al. (2009) afirmam que durante um processo fermentativo as quantidades de massa micelial e polissacarídeos não são necessariamente proporcionais, sendo dependentes dos diferentes fatores utilizados no cultivo.

Franco et al. (2004) encontraram rendimento de quitina de $23,8 \%$ e quitosana de $7,8 \%$. Franco et al. (2005) obtiveram $28 \%$ e $7,8 \%$ de quitina e quitosana, respectivamente. Stamford et al. (2007), relataram alto rendimento de quitina, $40,09 \%$, contudo, o rendimento de quitosana permaneceu dentro da média, 5,89\%. Um rendimento acima da média foi relatado por Santos et al. (2013) ao obter 2,14 $\mathrm{g} \mathrm{L}^{-1}$, e aproximadamente $13,8 \%$, de quitosana.

Todos os autores citados acima utilizaram cepas de C. elegans, as diferenças encontradas nos rendimentos de quitina e quitosana entre os estudos pode ser devido aos diferentes meios de cultivo, visto que Franco et al. (2004) utilizaram meio sintético para mucolares descrito por Andrade et al. (2000) e Franco et al. (2005) enriqueceu o respectivo meio com glicose, asparagina e tiamina. Ambos estudos obtiveram rendimentos próximos ao do presente trabalho.

Já Stamford et al. (2007) e Santos et al. (2013) utilizaram meios não comerciais a base de Jacatupé (Pachyrhizus erosus L. Urban) e a base de milhocina, respectivamente, relatando rendimentos expressivos.

Baixo rendimento de quitosana, $1,70 \%$, foi obtido por Amorim et al. (2006) ao cultivar Cunninghamella bertholletiae em meio YPD. Os mesmos relatam que a produção máxima de quitosana ocorreu nos três primeiros dias de crescimento, declinando após este período.

Stamford et al. (2007) também relataram que a maior produção de quitosana ocorreu nos primeiros dias de crescimento micelial. Os mesmos citam que rendimentos mais elevados de quitosana em estágios iniciais sugerem que a quitina-desacetilase, enzima que converte quitina em quitosana, prevaleceu nestas fases e que o fato da quitina nos estágios iniciais ser menos cristalina a torna mais susceptível à referida enzima.

A titulação potenciométrica apresenta duas etapas de dissociação, sendo a primeira etapa de neutralização do ácido presente, e a segunda etapa de neutralização de prótons dos grupos amino da quitosana. Essas etapas de dissociação formam pontos de inflexão que possibilitou calcular o grau de desacetilação da quitosana, correspondendo a $83,7 \%$ para a quitosana extraída do isolado UFT Ce09 e $80,5 \%$ para quitosana proveniente da cepa referência de C. elegans.

A quitosana obtida a partir de fungos da ordem Mucorales possuem grau de desacetilação variando

Tabela 1 - Biomassa seca das cepas isoladas de Cunninghamella sp. e cepa referência (CBMAI 0843) de C. elegans a cada 24 horas de cultivo em meio YPD.

\begin{tabular}{cccccc}
\hline \multirow{2}{*}{ Cepas } & \multicolumn{5}{c}{ Biomassa seca $\left(\mathrm{g} . \mathrm{L}^{-1}\right)$} \\
\cline { 2 - 6 } & $\mathbf{2 4}$ horas & $\mathbf{4 8}$ horas & $\mathbf{7 2}$ horas & $\mathbf{9 6}$ horas & $\mathbf{1 2 0}$ horas \\
\hline CBMAI 0843 & $7,29 \mathrm{a}$ & $10,78 \mathrm{c}$ & $9,80 \mathrm{a}$ & $9,07 \mathrm{c}$ & $7,62 \mathrm{c}$ \\
UFT Ce02 & $8,52 \mathrm{a}$ & $9,42 \mathrm{~d}$ & $9,35 \mathrm{a}$ & $8,09 \mathrm{c}$ & $4,69 \mathrm{~d}$ \\
UFT Ce04 & $5,07 \mathrm{a}$ & $12,20 \mathrm{~b}$ & $13,26 \mathrm{a}$ & $11,02 \mathrm{~b}$ & $10,87 \mathrm{a}$ \\
UFT Ce05 & $8,39 \mathrm{a}$ & $9,15 \mathrm{~d}$ & $8,69 \mathrm{a}$ & $8,07 \mathrm{c}$ & $5,68 \mathrm{~d}$ \\
UFT Ce06 & $7,52 \mathrm{a}$ & $9,36 \mathrm{~d}$ & $10,81 \mathrm{a}$ & $11,17 \mathrm{~b}$ & $8,41 \mathrm{~b}$ \\
UFT Ce08 & $8,52 \mathrm{a}$ & $20,17 \mathrm{a}$ & $12,24 \mathrm{a}$ & $13,15 \mathrm{a}$ & $8,99 \mathrm{~b}$ \\
UFT Ce09 & $8,95 \mathrm{a}$ & $9,34 \mathrm{~d}$ & $8,50 \mathrm{a}$ & $6,98 \mathrm{c}$ & $7,07 \mathrm{c}$ \\
UFT Ce11 & $9,73 \mathrm{a}$ & $10,72 \mathrm{c}$ & $8,40 \mathrm{a}$ & $9,31 \mathrm{c}$ & $4,88 \mathrm{~d}$ \\
UFT Ce14 & $7,13 \mathrm{a}$ & $10,36 \mathrm{c}$ & $10,50 \mathrm{a}$ & $8,03 \mathrm{c}$ & $7,61 \mathrm{c}$ \\
UFT Ce20 & $8,33 \mathrm{a}$ & $12,64 \mathrm{~b}$ & $11,83 \mathrm{a}$ & $10,30 \mathrm{~b}$ & $8,65 \mathrm{~b}$ \\
CV (\%) & 8,47 & 4,83 & 11,06 & 6,17 & 6,13 \\
\hline
\end{tabular}

${ }^{1}$ Médias seguidas de mesma letra minúscula, nas colunas, não diferem entre si pelo teste de Scott-Knott a $5 \%$ de significância. ${ }^{2} \mathrm{CV}=$ Coeficiente de variação.

Fonte: Autores. 
Tabela 2 - Rendimento de quitina e quitosana por cepas isoladas de fungo Cunninghamella sp. e pela cepa CBMAI 0843 de C. elegans cultivados em meio YPD.

\begin{tabular}{ccc}
\hline Cepas & $\begin{array}{c}\text { Rendimento de } \\
\text { Quitina (\%) }\end{array}$ & $\begin{array}{c}\text { Rendimento de } \\
\text { Quitosana (\%) }\end{array}$ \\
\hline CBMAI 0843 & 24,29 & 1,89 \\
UFT Ce02 & 21,42 & 2,69 \\
UFT Ce04 & 18,54 & 1,66 \\
UFT Ce05 & 22,90 & 2,43 \\
UFT Ce06 & 15,64 & 0,94 \\
UFT Ce08 & 30,28 & 4,66 \\
UFT Ce09 & 16,02 & 7,43 \\
UFT Ce11 & 30,33 & 5,91 \\
UFT Ce14 & 16,69 & 4,26 \\
UFT Ce20 & 22,38 & 5,55 \\
\hline
\end{tabular}

Fonte: Autores.

entre 80 e 90\% (AMORIM et al., 2006; BERGER et al., 2014; CHATTERJEE, 2005; FRANCO et al., 2005; POCHANAVANICH; SUNTORNSUK, 2002; STAMFORD et al., 2007).

O grau de desacetilação fornece um importante parâmetro relacionado a várias propriedades físicoquímicas e biológicas incluindo, solubilidade, viscosidade, habilidade de formar filmes, quelar metais, características estruturais e ópticas, assim como cristalinidade e hidrofilicidade (GAVHANE; GURAV; YADAV, 2013).

A quitosana com alto grau de desacetilação possui maior presença de grupos amínicos livres em sua estrutura, estes grupos conferem solubilidade em uma faixa específica de $\mathrm{pH}$, em solventes como ácidos orgânicos diluídos (acético e fórmico) e ácidos inorgânicos, originando soluções viscosas (DAMIAN et al., 2005).

As pesquisas científicas e as indústrias biotecnológicas têm optado por quitosana com alto grau de desacetilação e baixa massa molar, por possuir melhor solubilidade em água, conferindo caráter homogêneo e menor viscosidade ao sistema, auxiliando na produção de derivados do polímero (BAUMANN; FAUST, 2001).

A viscosidade da quitosana é diretamente proporcional à massa molar. Quitosana de baixa viscosidade exibe baixa massa molar, o que aumenta a solubilidade do polímero em água facilitando aplicações nas áreas alimentícias, médicas e na agricultura (BERGER et al., 2014; NIEDERHOFER; MÜLLER, 2004).
Em relação à massa molar da quitosana, a mesma foi determinada pelo parâmetro viscosidade através do tempo médio de escoamento das soluções de quitosana. A viscosimetria, embora seja um método não absoluto, é um dos processos mais utilizados para a determinação da massa molar de polímeros (SANTOS et al., 2003).

A viscosidade intrínseca, [ $\eta$ ], foi determinada pela extrapolação dos dados de viscosidade à diluição infinita, como mostram a Figura 1. As retas obtidas apresentaram excelentes coeficientes de correlação entre os pontos experimentais $(r>0,9)$.

Utilizando equação de Mark-Houwink-Sakurada obteve-se a massa molar da quitosana extraída do isolado UFT Ce09 de $43.031 \mathrm{~g} \mathrm{~mol}^{-1}$. Para a quitosana extraída da cepa referência, obteve-se a massa molar de $19.215 \mathrm{~g} \mathrm{~mol}^{-1}$, menor massa molar que a quitosana obtida do isolado UFT Ce09.

Segundo Roberts (1992), condições muito severas de reação, empregadas na desacetilação da quitina por fusão alcalina, e o emprego de soluções alcalinas concentradas, por tempos prolongados e a temperaturas elevadas, aumentam a possibilidade de obter quitosana de massa molar baixa.

Ao determinar a massa molar viscosimétrica média da quitosana comercial das marcas Aldrich ${ }^{\circledR}$ e Fluka ${ }^{\circledR}$, Santos et al. (2003) verificaram massa molar de 69.000 $\mathrm{g} \mathrm{mol}^{-1}$ e $252.000 \mathrm{~g} \mathrm{~mol}^{-1}$, respectivamente, valores acima do encontrado no presente estudo. Já Baumann e Faust (2001) após hidrolisar a quitosana com anidrido acético obtiveram massa molar de $29.000 \mathrm{~g} \mathrm{~mol}^{-1}$.

Quitosana com massa molar de 27.200 g.mol ${ }^{-1}$ extraída de C. elegans (UCP 542) cultivado em caldo a base de Jacatupé foi relatado por Stamford et al. (2007), resultado próximo ao do presente estudo. Já a quitosana extraída também de C. elegans (UCP/WFCC 0542) cultivado em meio composto por melaço de cana e milhocina por Berger et al. (2014) apresentou uma baixa massa molar 5.080 g.mol ${ }^{-1}$.

A análise final por espectrometria de infravermelho se apresenta como uma técnica imprescindível na caracterização de polissacarídeos como a quitosana, possibilitando classificar as principais bandas referentes aos grupos funcionais de sua estrutura. A Figura 2 apresenta os espectros obtidos a partir da quitosana extraída das cepas de Cunninghamella.

Os espectros de absorção infravermelho (Figura 2) apresentaram inicialmente uma banda intensa entre 3.300 a $3.430 \mathrm{~cm}^{-1}$ referente ao estiramento axial da ligação $\mathrm{O}-\mathrm{H}$, uma deformação angular da amina $\mathrm{N}-\mathrm{H}$ próximo a $1.590 \mathrm{~cm}^{-1}$, um estiramento axial do grupamento amida 
Figura 1 - Curvas de viscosidade reduzida versus concentração da quitosana do isolado UFT Ce09 de Cunninghamella sp. (๘) e da quitosana da cepa referência C. elegans (CBMAI 0843) (•) solubilizadas em tampão $\mathrm{NaAc} / \mathrm{HAc}, \mathrm{pH}=4,5$.

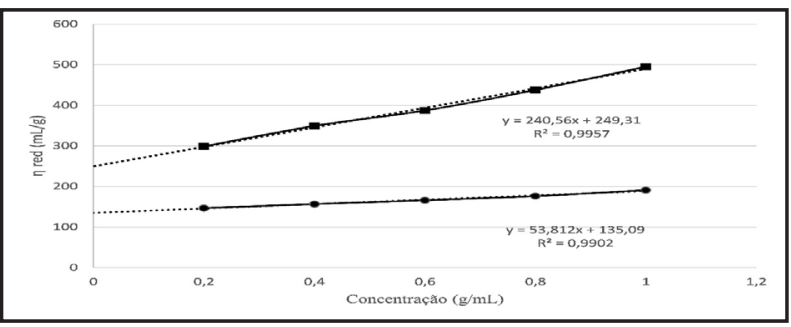

Fonte: Autores.

C-N em $1.420 \mathrm{~cm}^{-1}$, melhor observado no espectro (A), um estiramento angular simétrico de $\mathrm{C}-\mathrm{H}$ em $1.380 \mathrm{~cm}^{-1}$ e um estiramento axial dos grupos amina $\mathrm{C}-\mathrm{N}$ entre $1.100-1.250 \mathrm{~cm}^{-1}$. As mesmas características espectrais da quitosana fúngica podem ser observadas no espectro da quitosana comercial demonstrado por Santos et al. (2013).

As deformações angulares da quitosana nos respectivos comprimentos de onda observados no presente estudo, também foram relatadas por diversos autores (FRANCO et al., 2005; STAMFORD et al., 2007; BERGER et al., 2014).

Figura 2 - Espectro de absorção infravermelho da quitosana obtida do isolado UFT Ce09 de Cunninghamella. sp. (A) e obtida da cepa referência $C$. elegans (CBMAI 0843) (B)
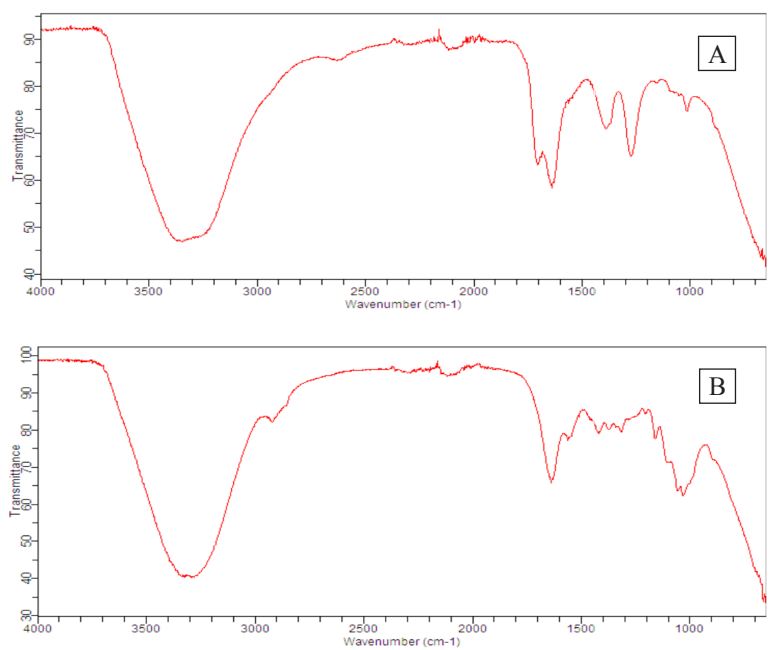

Fonte: Autores.
Figura 3 - Diferenciação estrutural da quitina e quitosana
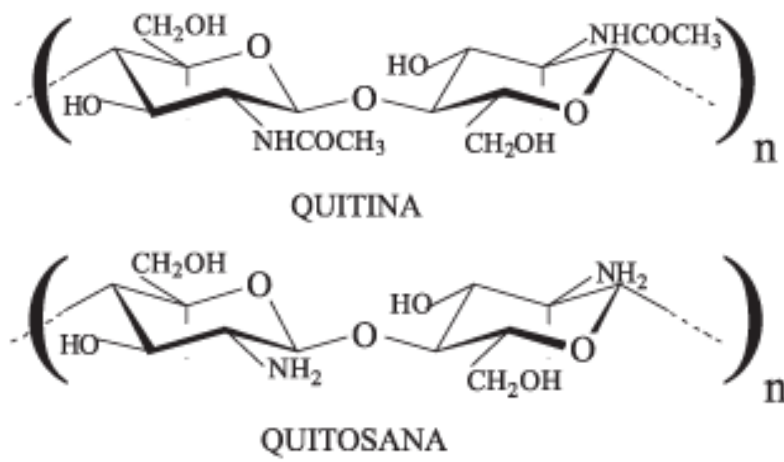

Fonte: Battisti e Campana-Filho (2008).

Portanto, a quitosana fúngica deste estudo apresentou propriedades que atestam sua qualidade e de grande interesse biotecnológico e comercial. A mesma torna-se uma alternativa para comercialização por meio de fontes não tradicionais. O fungo Cunninghamella sp. pode ser considerado uma fonte alternativa promissora para a extração de quitina e quitosana, estimulando a indústria biotecnológica com base nos métodos avançados de fermentação.

\section{Conclusões}

As cepas isoladas apresentaram bom crescimento micelial, com destaque ao isolado UFT $\mathrm{Ce} 08$ que apresentou o maior valor de biomassa seca, em comparação aos outros isolados.

O isolado UFT Ce 11 apresentou o maior quantitativo de quitina e o isolado UFT Ce09 o maior quantitativo de quitosana. A maioria do isolados avaliados produziram mais quitosana que a cepa referência.

O grau de desacetilação apresentou-se dentro dos limites especificados na literatura, sendo $83,7 \%$ para quitosana obtida do isolado UFT Ce09 e $80,5 \%$ para quitosana obtida da cepa referência. As massas molares determinadas através da viscosidade para a quitosana do isolado UFT Ce09 e da cepa referência foram de 43.031 g.mol ${ }^{-1}$ e de 19.215 g.mol ${ }^{-1}$, respetivamente. Esses valores caracterizam a quitosana do presente estudo com massa molar baixa. A espectrometria de infravermelho apresentou bandas com comprimentos de onda e grupos funcionais coincidentes com a literatura e com a quitosana comercial. 
Lopes, J. C. et al.

\section{Referências}

ABREU, F. R.; CAMPANA-FILHO, S. P. Preparation and characterization of carboxymethylchitosan. Polímeros, São Carlos, v. 15, n. 2, p. 79-83, 2005.

ALEXOPOULOS, C. J.; MIMS, C. W.; BLACKWELL, M. Introductory mycology. 4. ed. New York: J. Wiley \& Sons, 1996.

AMORIM, R. V. S.; PEDROSA, R. P.; FUKUSHIMA, K.; MARTÍNEZ, C. R.; LEDINGHAM, W. M.; CAMPOS-TAKAKI, G. M. Alternative carbon sources from sugar cane process for submerged cultivation of Cunninghamella bertholletiae to produce chitosan. Food Technology and Biotechnology, Bethesda, v. 44, n. 4, p. 519-523, 2006.

ANDRADE, V. S.; BARROS NETO, B.; SOUZA, W.; CAMPOS-TAKAKI, G. M. A factorial design analysis of chitin production by Cunninghamella elegans. Canadian Journal of Microbiology, Bethesda, v. 46, p. 1042-1045, 2000.

BARNETT, H. L.; HUNTER, B. Illustrated genera of imperfect fungi. Minnesota: Burgess Publishing Company, 1999.

BATTISTI, M. V.; CAMPANA-FILHO, S. P. Obtenção e caracterização de $\alpha$-quitina e quitosanas de cascas de Macrobrachium rosembergii. Química Nova, São Paulo, v. 31, n. 8, p. 2014-2019, 2008.

BAUMANN, H.; FAUST, V. Concepts for improved regioselective placement of O-sulfo, $\mathrm{N}$-sulfo, $\mathrm{N}$-acetyl, and $\mathrm{N}$-carboxymethyl groups in chitosan derivatives. Carbohydrate Research, Amsterdam, v. 331, n. 1, p. 43-57, 2001.

BERGER, L. R. R.; FELIX, F. F.; BORGES, T. K. S.; MALHEIROS, S. M. M.; STAMFORD, N. P. Cultivo de Cunningamella Elegans (UCP 542) em meio BD. In: JORNADA DE ENSINO PESQUISA E EXTENSÃO DA UNIVERSIDADE FEDERAL DE PERNAMBUCO, 9., 2009, Recife. Anais... Recife: UFRPE, 2009. CD-Rom.

BERGER, L. R. R.; STAMFORD, N. P.; WILLADINO, L. G.; LARANJEIRA, D.; DE LIMA, M. A. B.; MALHEIROS, S. M. M.; STAMFORD, T. C. M. Cowpea resistance induced against Fusarium oxysporum f. sp. tracheiphilum by crustaceous chitosan and by biomass and chitosan obtained from Cunninghamella elegans. Biological Control, Orlando, v. 92 , p. $45-54,2016$.
BERGER， L. R. R.; STAMFORD, T. C. M.; STAMFORD, T. M.; ALCÂNTARA, S. R. C.; SILVA, A. C.; SILVA, A. M.; CAMPOS-TAKAKI, G. M. Green conversion of agroindustrial wastes into chitin and chitosan by Rhizopus arrhizus and Cunninghamella elegans strain. International Journal of Molecular Sciences, Basel, v. 15, n. 5, p. 9082-9102, 2014.

CAMPANA-FILHO, S. P.; BRITTO, D.; CURTI, E.; ABREU, F. R.; CARDOSO, M. B.; BATTISTI, M. V.; LAVALL, R. L. Extração, estruturas e propriedades de alfa- e beta-quitina. Química Nova, São Paulo, v. 30, n. 3, p. 644-650, 2007.

CAMPBELL, C. K.; JOHNSON, E. M. Identification of pathogenic fungi. Nova Jersey: J. Wiley \& Sons, 2013.

CHATTERJEE, S. Chitosan from Mucor rouxii: production and physico-chemical characterization. Process Biochemistry, London, v. 40, p. 395-400, 2005.

CHIEN, R. C.; YEN, M. T.; MAU, J. L. Antimicrobial and antitumor activities of chitosan from shiitake stipes, compared to commercial chitosan from crab shells. Carbohydrate Polymers, Worcester, v. 138, p. 259-264, 2016.

CROFTON, A. R.; HUDSON, S. M.; HOWARD, K.; PENDER, T.; ABDELGAWAD, A.; WOLSKI, D.; KIRSCH, W. M. Formulation and characterization of a plasma sterilized, pharmaceutical grade chitosan powder. Carbohydrate Polymers, Worcester, v. 146, p. 420-426, 2016.

CZECHOWSKA-BISKUP, R.; JAROSIŃSKA, D.; ROKITA, B.; ULAŃSKI, P.; ROSIAK, J. M. Determination of degree of deacetylation of chitosancomparision of methods. Progress on Chemistry and Application of Chitin and Its Derivates, Warsaw, v. 17, p. 5-20, 2012.

DAMIAN, C.; BEIRÃO, L. H.; FRANCISCO, A. D.; ESPIRITO SANTO, M. L. P.; TEIXEIRA, E. Quitosana: um amino polissacarídio com características funcionais. Alimentos e Nutrição, Araraquara, v. 16, n. 2, p. 195-205, 2005.

DUDDINGTON, C. L. The ecology of predacious fungi i. preliminary survey. Transactions British Mycological Society, London, v. 34, p. 322-331, 1951. 
FRÁGUAS, R. M.; SIMÃO, A. A.; FARIA, P. V.; QUEIROZ, E. D. R.; OLIVEIRA JUNIOR, Ê. N. D.; ABREU, C. M. P. D. Preparation and characterization chitosan edible films. Polímeros, São Carlos, v. 25, p. 48-53, 2015.

FRANCO, L. O.; MAIA, R. D. C. C.; PORTO, A. L. F.; MESSIAS, A. S.; FUKUSHIMA, K.; CAMPOSTAKAKI, G. M. D. Heavy metal biosorption by chitin and chitosan isolated from Cunninghamella elegans (IFM 46109). Brazilian Journal of Microbiology, São Paulo, v. 35, n. 3, p. 243-247, 2004.

FRANCO, L. O.; STAMFORD, T. C. M.; STAMFORD, N. P.; CAMPOS-TAKAKI, G. M. Cunninghamella elegans (IFM 46109) como fonte de Quitina e Quitosana. Revista Analytica, Rio de Janeiro, v. 4, p. 40-44, 2005.

FREITAS, S. M. C.; STAMFORD, T. C. M.; FRANCO, L. O.; CAMPOS-TAKAKI, G. M. Effect of salinity and glucose on chitin and chitosan production by Cunninghamella elegans. Asian Chitin Journal, Allahabad, v. 2, p. 29-38, 2006.

FUKUDA, E. K.; VASCONCELOS, A. F. D.; MATIAS, A. C.; BARBOSA, A. M.; DEKKER, R. F. H.; SILVA, M. D. L. C. Polissacarídeos de parede celular fúngica: purificação e caracterização. Semina: Ciências Agrárias, Londrina, p. 117-134, 2009.

GAVHANE, Y. N.; GURAV, A. S.; YADAV, A. V. Chitosan and Its Applications: A Review of Literature. Internacional Journal of Research in Pharmaceutical Biomedical Science, Pakistan, v. 4, p. 311-331, 2013.

KARIMI, K.; ZAMANI, A. Mucor indicus: biology and industrial application perspectives: A review. Biotechnology Advances, New York, v. 31, n. 4, p. 466-481, 2013.

KASAAI, M. R. Calculation of Mark-HouwinkSakurada (MHS) equation viscometric constants for chitosan in any solvent-temperature system using experimental reported viscometric constants data. Carbohydrate Polymers, Barking, v. 68, n. 3, p. 477488, 2007.

NIEDERHOFER, A.; MÜLLER, B. W. A method for direct preparation of chitosan with low molecular weight from fungi. European Journal of Pharmaceutics and Biopharmaceutics, London, v. 57, n. 1, p. 101105, 2004.
PINTO, Z. V.; BETTIOL, W.; MORANDI, M. A. B. Efeito de casca de camarão, hidrolisado de peixe e quitosana no controle da murcha de Fusarium oxysporum f. sp. chrysanthemi em crisântemo. Tropical Plant Pathology, Brasília, v. 35, n. 1, p. 16-23, 2010.

PITT, J. I.; HOCKING, A. D. Zygomycetes. In: Fungi and food spoilage. New York: Springer, 2009. p. 145168.

POCHANAVANICH, P.; SUNTORNSUK, W. Fungal chitosan production and its characterization. Letters in Applied Microbiology, Oxford, v. 35, n. 1, p. 17-21, 2002.

ROBERTS, G. A. F. Chitin chemistry. London: The Mucmillan Press, 1992.

SANTOS, E. R.; SILVA, M. C. F.; SOUZA, P. M.; SILVA, A. C.; PAIVA, S. C.; ALBUQUERQUE, C. D.; CAMPOS-TAKAKI, G. M. Enhancement of Cunninghamella elegans UCP/WFCC 0542 biomass and chitosan with amino acid supply. Molecules, Basel, v. 18, n. 9, p. 10095-10107, 2013.

SANTOS, J. E.; SOARES, J. P.; DOCKAL, E. R.; FILHO, S. P. C.; CAVALHEIRO, É. T. Caracterização de quitosanas comerciais de diferentes origens. Polímeros, São Carlos, v. 13, n. 4, p. 242-249, 2003.

SANTOS, M. A.; FERRAZ, S.; MUCHOVEJ, J. J. Detection and ecology of nematophagous fungi from Brazilian soils. Nematologia Brasileira, Campinas, v. 15, p. 121-134, 1991.

SILVA, H. S. R. C.; SANTOS, K. S. C. R.; FERREIRA, E. I. Quitosana: derivados hidrossolúveis, aplicações farmacêuticas e avanços. Química Nova, São Paulo, v. 29, n. 4, p. 776-785, 2006.

SINGLA, A. K.; CHAWLA, M. Chitosan: Some pharmaceutical and biological aspects - an update. Journal of Pharmacy and Pharmacology, London, v. 53, n. 8, p. 1047-1067, 2001.

SOUZA, P. M. Caracterização bioquímica, fisiológica e ultraestrutural do processo de biossorção do cobre por Cunninghamella elegans - UCP 542. 2004. 67 f. Dissertação (Mestrado em Biologia de Fungos) Centro de Ciências Biológicas, Universidade Federal de Pernambuco, Recife, 2004. 
STAMFORD, T. C. M.; STAMFORD, T. L. M.; STAMFORD, N. P.; BARROS NETO, B. D.; CAMPOSTAKAKI, G. M. D. Growth of Cunninghamella elegans UCP 542 and production of chitin and chitosan using yam bean medium. Electronic Journal of Biotechnology, Valparaíso, v. 10, n. 1, p. 61-68, 2007.

TAYEL, A. A.; GHARIEB, M. M.; ZAKI, H. R.; ELGUINDY, N. M. Bio-clarification of water from heavy metals and microbial effluence using fungal chitosan. International Journal of Biological Macromolecules, Guildford, v. 83, p. 277-281, 2016.

THARANATHAN, R. N.; KITTURE, F. S. Chitin-the undisputed biomolecule of great potential. Critical Reviews in Food Science and Nutrition, Boca Raton, v. 43, p. 61-87, 2003.

VÁZQUEZ， J. A.; RODRÍGUEZ-AMADO， I.; MONTEMAYOR, M. I.; FRAGUAS, J.; GONZÁLEZ, M. D. P.; MURADO, M. A. Chondroitin sulfate, hyaluronic acid and chitin/chitosan production using marine waste sources: Characteristics, applications and eco-friendly processes: A review. Marine Drugs, Basel, v. 11, n. 3, p. 747-774, 2013.

ZAMANI, A. Superabsorbent polymers from the cell wall of zygomycetes fungi. 2010. $60 \mathrm{f}$. $\mathrm{PhD}$ Thesis (Chemical and Biological Engineering) - Chalmers University of Technology, Göteborg, 2010. 